\title{
Impact of magnetization easy-axis distributions on the ferromagnet-antiferromagnet exchange-coupling estimation
}

\author{
J. Geshev, ${ }^{1,2}$ S. Nicolodi, ${ }^{1}$ L. G. Pereira, ${ }^{1}$ J. E. Schmidt, ${ }^{1}$ V. Skumryev, ${ }^{2,3}$ S. Suriñach, ${ }^{2}$ and M. D. Baró ${ }^{2}$ \\ ${ }^{1}$ Instituto de Física, Universidade Federal do Rio Grande do Sul, Porto Alegre, 91501-970 Rio Grande do Sul, Brazil \\ ${ }^{2}$ Departament de Física, Universitat Autónoma de Barcelona, Bellaterra, 08193 Barcelona, Spain \\ ${ }^{3}$ Institut Català de Recerca i Estudis Avançats (ICREA), 08010 Barcelona, Spain
}

(Received 16 January 2008; revised manuscript received 14 March 2008; published 17 April 2008)

\begin{abstract}
It is frequently reported that different measurement techniques may yield distinct values for the exchange anisotropy, being the difference of up to one order of magnitude on comparing magnetization loop with ac susceptibility measurements. We show, by both model calculations and a straight-forward experiment, that at least part of this difference could be attributed to big errors in the evaluation of the ferromagnet/ antiferromagnet exchange coupling strengths obtained by means of ac magnetic susceptibility and dc magnetometry measurements which are caused by neglecting the easy-axis distributions or underestimating the uniaxial anisotropy of the ferromagnetic material.
\end{abstract}

DOI: 10.1103/PhysRevB.77.132407

PACS number(s): 75.30.Gw, 75.30.Cr, 71.70.Gm, 75.60.-d

The exchange-bias (EB) phenomenon, ${ }^{1,2}$ although already in use in magnetoelectronic devices, continues to attract significant attention owing to its captivating physics and also due to several controversial issues concerning the coupling between ferromagnetic (FM) and antiferromagnetic (AF) materials. Among them is the fact that different measurement techniques may yield distinct values for the exchange anisotropy. $^{3-15}$ This has been attributed to twisting of the magnetization through the thickness of the FM film, ${ }^{3}$ inconsistency of the model used to interpret the experiment, ${ }^{12,14}$ or different numbers of stable AF moments at the FM/AF interface in low and high field measurements. ${ }^{11,14}$ The EB is frequency dependent as well; e.g., the ferromagnetic resonance (FMR) fields (used in the definition of the respective EB field), which are obtained at two different excitation frequencies, could differ considerably. ${ }^{16}$ Reviews on EB can be found, e.g., in Refs. 17-19.

In thin-film systems, the exchange-coupling field $H_{E}$ is usually defined as $J_{E} /\left(t_{F M} M_{F M}\right)$, where $J_{E}$ is the $\mathrm{FM} / \mathrm{AF}$ exchange-coupling constant and $t_{F M}$ is the thickness of the FM layer with saturation magnetization $M_{F M}$ and anisotropy constant $K_{F M}$. It is worth emphasizing that $H_{E}$ is not the same as the exchange-bias field $H_{e b}$. In a dc magnetization measurement, $H_{e b}$ is defined as the hysteresis loop's field shift, $H_{e b}^{\mathrm{dc}}$; its definition in a FMR measurement, $H_{e b}^{\mathrm{FMR}}$, could be found in Ref. 13, for example. In general, ${ }^{12} H_{e b}$ depends also on $K_{F M}$ and on the AF anisotropy constant $K_{A F}$.

It was shown that the EB fields, derived by means of reversible (e.g., FMR, ac susceptibility) and irreversible (magnetization curves, torque) techniques, must, in general, be different. ${ }^{13,14}$ However, even when $H_{e b}^{\mathrm{FMR}}$ and $H_{e b}^{\mathrm{dc}}$ coincide, ${ }^{14}$ the values of the exchange anisotropy, derived from the corresponding model calculations, $J_{E}^{\mathrm{FMR}}$ and $J_{E}^{\mathrm{dc}}$, could be different. Some of the moments, stable in a FMR measurement, are unstable in a magnetization curve trace, since the measurement time of the latter is much longer than the FMR relaxation time, thus explaining why $J_{E}^{\mathrm{FMR}}>J_{E}^{\mathrm{dc}}$.

Very recently, Åkerman et al. ${ }^{15}$ reported up to eight times larger values of the exchange anisotropy estimated from transverse ac magnetic susceptibility $\left(J_{E}^{\text {ac }}\right)$ than those ob- tained from hysteresis measurements and stated that the susceptibility data give the correct measures of the exchange anisotropy. In such measurements, however, there is no ana$\log$ to $H_{e b}$ and $J_{E}^{\mathrm{ac}}$ is obtained by comparing the experimental data with those calculated in the framework of a proper phenomenological model. ${ }^{4,9,15}$ The accuracy with which the anisotropy parameters are determined relies on this model, and when the magnetic free energy density is not correctly described, this method can lead to substantial errors. ${ }^{20}$ The same, in general, holds also for dc magnetometry.

In this Brief Report, we show, by means of both model simulations and a simple experiment, that the FM/AF exchange-coupling strengths, obtained through interpretation of magnetic susceptibility and dc magnetometry measurements, could differ substantially due to neglecting the FM and/or AF easy-axes distributions or underestimating the FM uniaxial anisotropy.

The first model discussed here is the one that allows a formation of a domain wall at the AF part of the FM/AF interface $^{21,12}$ and has the FM uniaxial anisotropy field $H_{U}$ $\left(=2 K_{F M} / M_{F M}\right)$, the AF domain-wall anisotropy field $H_{W}$ $\left[=\sigma_{W} /\left(t_{F M} M_{F M}\right)\right.$, where $\sigma_{W}$ is the energy per unit surface of a $90^{\circ} \mathrm{AF}$ domain wall], and $H_{E}$ as parameters. Considering in-plane FM and AF easy axes and also external magnetic field $\mathbf{H}$ applied in the film's plane, the free energy per unit area can be written as

$$
\begin{aligned}
E_{\mathrm{DWF}}= & -\mathbf{H} \cdot \mathbf{M}_{\mathbf{F M}} t_{F M}-K_{F M} t_{F M}\left(\frac{\mathbf{M}_{\mathbf{F M}} \cdot \hat{\mathbf{u}}_{\mathbf{F M}}}{M_{F M}}\right)^{2} \\
& -J_{E} \frac{\mathbf{M}_{\mathbf{F M}} \cdot \mathbf{M}_{\mathbf{A F}}}{M_{F M} M_{A F}}-\sigma_{W} \frac{\mathbf{M}_{\mathbf{A F}} \cdot \hat{\mathbf{u}}_{\mathbf{A F}}}{M_{A F}}
\end{aligned}
$$

The first three terms are the Zeeman, FM uniaxial, and FM/AF exchange anisotropy energies, respectively, and the last term is the AF anisotropy; the unit vectors $\hat{\mathbf{u}}_{\mathbf{F M}}$ and $\hat{\mathbf{u}}_{\mathbf{A F}}$ represent the FM and AF uniaxial anisotropy directions, respectively. Henceforth, we refer to this model as the domainwall formation (DWF) model. 
When the last term in Eq. (1) is substituted by uniaxial AF anisotropy term, the DWF model becomes that of Meiklejohn. ${ }^{2}$ Moreover, if the value of the AF anisotropy (or $\sigma_{W}$ in the DWF model) is very high, then $\mathbf{M}_{\mathbf{A F}}$ will always point along $\hat{\mathbf{u}}_{\mathrm{AF}}$ and both Meiklejohn's and DWF models are reduced to one with energy expression,

$$
\begin{aligned}
E_{\mathrm{RAF}}= & -\mathbf{H} \cdot \mathbf{M}_{\mathbf{F M}} t_{F M}-K_{F M} t_{F M}\left(\frac{\mathbf{M}_{\mathbf{F M}} \cdot \hat{\mathbf{u}}_{\mathbf{F M}}}{M_{F M}}\right)^{2} \\
& -J_{E} \frac{\mathbf{M}_{\mathbf{F M}} \cdot \hat{\mathbf{u}}_{\mathbf{A F}}}{M_{F M}},
\end{aligned}
$$

abbreviated here as RAF (rigid antiferromagnetic moment) model which, despite its simplicity, seems to be appropriate for some EB systems ${ }^{22}$ and is, sometimes, used for a broad temperature range. ${ }^{4,6,15}$ It must be stressed, however, that the RAF model could be eventually applied for interpretation of data obtained at temperatures much below the AF blocking temperature $T_{B}$; in vicinity of $T_{B}, K_{A F}$ vanishes and $\mathbf{M}_{\mathbf{A F}}$ is rotated away from its easy axis thus invalidating the model. Therefore, the more general and flexible DWF model should be used in order to interpret data obtained at temperatures close to $T_{B}$.

The transverse biased ac susceptibility $\chi_{t}$ is a high sensitivity technique which gives the response of the system to a small ac magnetic field applied perpendicular to a dc bias field $H_{\mathrm{dc}}$. The characteristic curve obtained when decreasing $H_{\mathrm{dc}}$ from saturation can be used ${ }^{23}$ to determine the effective anisotropy field $H_{\text {eff. }}$. The expression for $\chi_{t}$ when $H_{\mathrm{dc}} \simeq 0$ could be derived following, e.g., the procedure used by Xi et al. ${ }^{9}$ which gives $\chi_{t}=M_{F M} / H_{\text {eff }}$, where

$$
H_{\text {eff }}^{\text {DWF }}=H_{U}+\frac{H_{E} H_{W}}{H_{E}+H_{W}}
$$

for the case of the DWF model, ${ }^{9,12}$ and

$$
H_{\text {eff }}^{\mathrm{RAF}}=H_{U}+H_{E}
$$

for the RAF one. ${ }^{9,15}$ The corresponding $H_{E}$ become

$$
\begin{gathered}
H_{E, \mathrm{DWF}}^{\mathrm{ac}}=\left(\frac{1}{\frac{M_{F M}}{\chi_{t}}-H_{U}}-\frac{1}{H_{W}}\right)^{-1}, \\
H_{E, \mathrm{RAF}}^{\mathrm{ac}}=\frac{M_{F M}}{\chi_{t}}-H_{U} .
\end{gathered}
$$

Making $H_{W}$ infinitely high, Eqs. (3) and (5) reduce to Eqs. (4) and (6), respectively, as expected.

The hysteresis loop shift field for intermediate values of $H_{W}$, does depend ${ }^{12}$ on $H_{U}$, contrary to the commonly accepted (valid, however, for very high $H_{W}$ values only) expression $H_{E}^{\mathrm{dc}}=H_{e b}^{\mathrm{dc}}$, while weak AF anisotropy corresponds to $H_{W}=H_{e b}^{\mathrm{dc}}$.

The dashed line in Fig. 1 represents the hysteresis loop obtained for the simplest case of an exchange-coupled FM/AF pair with $\hat{\mathbf{u}}_{\mathrm{FM}}\left\|\hat{\mathbf{u}}_{\mathrm{AF}}\right\| \mathbf{H}_{\mathrm{dc}}$, calculated through the DWF model using $H_{E}=25 \mathrm{Oe}, H_{U}=100 \mathrm{Oe}$, and $H_{W}$ $=500$ Oe. Let us assume that this is an experimentally mea-

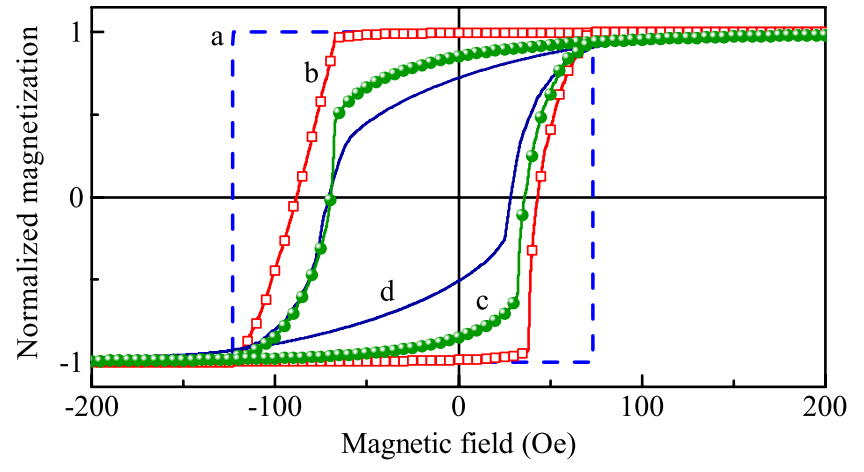

FIG. 1. (Color online) Easy-axis hysteresis loops for a FM/AF bilayer simulated using $H_{U}=100 \mathrm{Oe}, H_{W}=500 \mathrm{Oe}$, and $H_{E}=25 \mathrm{Oe}$. (a) The dashed curve represents a single FM/AF pair $\left(H_{e b}^{\mathrm{dc}}=25 \mathrm{Oe}\right.$, $H_{c}=98 \mathrm{Oe}, M_{F M} / \chi_{t}=124 \mathrm{Oe}$ ); (b) empty symbols correspond to a single FM domain and equally distributed in-plane AF easy axes with $55^{\circ}$ maximum deviation away from the FM easy direction $\left(H_{e b}^{\mathrm{dc}}=23 \mathrm{Oe}, H_{c}=66 \mathrm{Oe}, M_{F M} / \chi_{t}=121 \mathrm{Oe}\right)$; (c) full symbols represent the case of coupled FM and AF easy-axis distributions with $55^{\circ}$ maximum deviation $\left(H_{e b}^{\mathrm{dc}}=17 \mathrm{Oe}, H_{c}=53 \mathrm{Oe}, M_{F M} / \chi_{t}=137 \mathrm{Oe}\right)$; and (d) the solid curve is calculated assuming randomly distributed FM easy axes and the AF ones with $55^{\circ}$ maximum deviation $\left(H_{e b}^{\mathrm{dc}}\right.$ $\left.=21 \mathrm{Oe}, H_{c}=50 \mathrm{Oe}, M_{F M} / \chi_{t}=202 \mathrm{Oe}\right)$.

sured curve characterized by $H_{e b}^{\mathrm{dc}}=25$ Oe and coercivity $H_{c}$ $=98 \mathrm{Oe}$, and that one measures $H_{\mathrm{eff}}=M_{F M} / \chi_{t} \simeq 124 \mathrm{Oe}$, the value that Eq. (3) would also give. Let us now suppose that one, using these data and the RAF model through Eq. (6), derives $H_{E}^{\text {ac }}$ [where $H_{U}$ should be determined independently since $M_{F M} / \chi_{t}\left(=H_{\text {eff }}\right)$ is a combination of anisotropy fields as seen from Eqs. (3) and (4)]. Assuming $H_{U}=H_{c}$, plausible here due to the rectangular shape of the loop, the resultant $H_{E}^{\text {ac }}$ equals $26 \mathrm{Oe}$, in a good agreement with $H_{e b}^{\mathrm{dc}}$ as expected since, when $H_{W} \gg H_{E}$, the DWF and RAF models coincide and $H_{e b}^{\mathrm{dc}}=H_{E}$, as mentioned above.

Rectangular easy-axis curves, however, are rarely obtained since real FM/AF bilayers are almost never epitaxially grown pairs of single crystals and normally there exists $\mathrm{AF}$ and/or FM easy axes texturing. Preferential AF easy-axis distribution reduces the coercivity and makes the magnetization curve more rounded, more notably when the applied field direction is close to the EB one. The $H_{e b}^{\mathrm{dc}}$ values, however, remain almost unaffected regardless of the field orientation. ${ }^{24}$

Hysteresis loops obtained for different easy-axis distributions are shown in Fig. 1 (details on the numerical procedure employed in their calculation can be found in Refs. 12-14). The AF easy-axis maximum deviation of $55^{\circ}$ assumed here is consistent with the fact that most of the AF materials used in EB systems have cubic anisotropy. Usually, in order to establish the EB direction, magnetic field of amplitude sufficient to align the FM moments is applied during the cooling process. At the measurement (low) temperature, the AF moments deviate away from the field direction toward their closest easy axes. For cubic-anisotropy materials, their distribution is confined to a solid angle, the half-apex angle of which is ${ }^{26} \cos ^{-1}(1 / \sqrt{3}) \simeq 55^{\circ}$. The $H_{e b}^{\mathrm{dc}}$ and $H_{c}$ values for each loop are given in the caption of Fig. 1 along with the corresponding values ${ }^{25}$ of $M_{F M} / \chi_{t}$.

We used the RAF model through Eq. (6) to derive $H_{E}^{\text {ac }}$ and 
compare it with $H_{e b}^{\mathrm{dc}}$ for the cases corresponding to the loops plotted in Fig. 1. Since the values of $M_{F M} / \chi_{t}$ increase and $H_{c}$ decrease as compared to those of the case of no distribution, the $H_{E, \mathrm{RAF}}^{\mathrm{ac}} / H_{e b}^{\mathrm{dc}}$ ratio significantly increases. Starting from 1.0 for case (a), it becomes 2.4, 4.9, and 7.2 for the cases (b), (c), and (d), respectively.

In these estimations, we employed $H_{U}=H_{c}$; however, since often in EB systems $H_{c}$ is enhanced in relation to the unbiased case, one could use the values of $H_{U}$ rather lower than $H_{c}$ in Eq. (6). This will further increase $H_{E}^{\mathrm{ac}} / H_{e b}^{\mathrm{dc}}$, e.g., assuming $40 \%$ lower $H_{U}$ than $H_{c}$ for case (d) results in a ratio of 8.1 .

It is possible that the FM and AF easy axes are not parallel, i.e., there is an (off-aligned) angle between them. Although off-aligned exchange coupling has rarely been reported, $4,15,27-29$ it is, in principle, not difficult to be produced. If the magnetic field during the cooling is applied in a direction different from $\hat{\mathbf{u}}_{\mathrm{FM}}$, the FM moments line up with $\mathbf{H}_{\mathrm{dc}}$ and, in their turn, align the spins of the cubic-anisotropy $\mathrm{AF}$ in this direction. If the starting temperature and the duration of the annealing are not appropriate to provoke such a reorientation in the FM, an off-aligned system is produced. Its $\chi_{t}$ could be evaluated using the expression derived by Åkerman et al. ${ }^{15}$ for the RAF model, and the resulting $H_{E}^{\text {ac }}$ is

$$
H_{E, \mathrm{RAF}}^{\text {ac,off-align }}=\frac{\frac{M_{F M}}{\chi_{t}} \cos ^{2} \theta-H_{U} \cos 2\left(\phi_{F M}-\theta\right)}{\cos \left(\theta-\phi_{A F}\right)},
$$

where $\theta, \phi_{F M}$, and $\phi_{A F}$ are the angles that $\mathbf{H}_{\mathrm{dc}}$ makes with $\mathbf{M}_{\mathrm{FM}}, \hat{\mathbf{u}}_{\mathrm{FM}}$, and $\hat{\mathbf{u}}_{\mathrm{AF}}$, respectively.

Since in the work of Åkerman et al. ${ }^{15}$ that reported $H_{E}^{\mathrm{ac}} / H_{e b}^{\mathrm{dc}}$ ratios of up to eight one finds no dc magnetometry data (hysteresis loops, $H_{e b}^{\mathrm{dc}}$, coercivity, etc.), explicitly given, we are not able to evaluate numerically the role of the easyaxis distribution for their specific case. However, we derived $H_{E}^{\mathrm{ac}}$ through Eq. (7) and compared it to $H_{e b}^{\mathrm{dc}}$ for a hypothetic off-aligned RAF system $\left(H_{W}=\infty\right)$ using the parameters from Fig. 1 for $\phi_{F M}=66^{\circ}$ (the same as in Ref. 15) and $\phi_{A F}=0^{\circ}$. Even in this simplest case of a single off-aligned FM/AF pair, the use of $H_{U}=71 \mathrm{Oe}\left(=H_{c}\right.$ of the easy-axis loop showing $\left.H_{e b}^{\mathrm{dc}}=39 \mathrm{Oe}\right)$ results in a significant error in the estimation of $H_{e b}^{\mathrm{ac}}$, being $H_{E}^{\mathrm{ac}} / H_{e b}^{\mathrm{dc}} \simeq 1.9$ instead of 1.0 .

With the help of Eq. (7) for temperatures higher than the blocking temperature $\left(H_{E, \mathrm{RAF}}^{\text {ac,off-align }}=0\right)$, Åkerman et al. ${ }^{15} \mathrm{ob}-$ tained very low FM anisotropy value and extrapolated it for the low-temperature region. They applied this procedure, valid for the single-domain case only, on a polycrystalline sample that showed weak texturing, i.e., broad FM easy-axis distribution, which certainly resulted in a significant underestimation of $H_{U}$. It turns out that the hysteresis loop for $\mathbf{H}_{\mathrm{dc}}$ applied at $-66^{\circ}$ with respect to $\hat{\mathbf{u}}_{\mathbf{F M}}$ is not very different from that of $24^{\circ}$ and the corresponding $M_{F M} / \chi_{t}$ is rather higher than that of a single FM domain. Such underestimation of $H_{U}$ leads to artificially high $H_{E, \mathrm{RAF}}^{\mathrm{ac}}$ at low temperatures since, in Eq. (7), $H_{U}$ is subtracted from the $M_{F M} / \chi_{t}$ term. The value of the latter is, in its turn, higher than that of a single-domain FM/AF pair owing to, once again, the non-

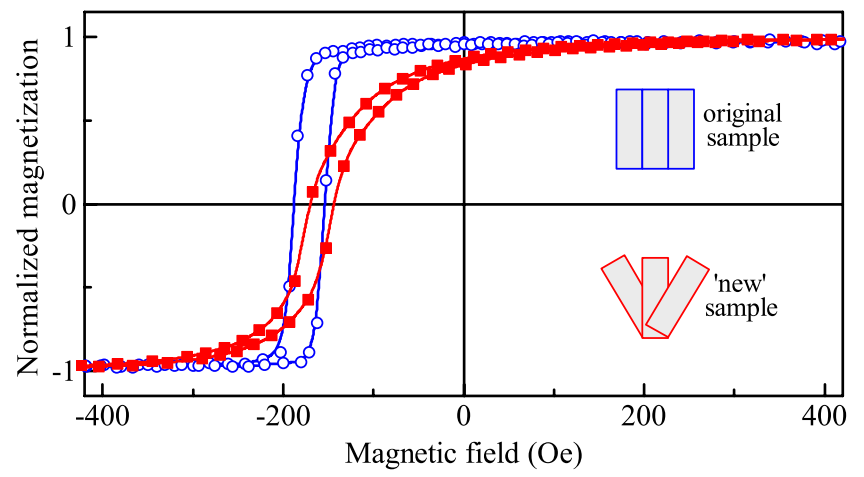

FIG. 2. (Color online) Easy-axis hysteresis loop of IrMn/Co film (Refs. 14 and 30) (empty circles) and the respective loop (full squares) measured after the sample was cut along its easy direction into three pieces and two of them set to form $+30^{\circ}$ and $-30^{\circ}$ with the other, as shown in the scheme.

rectangular hysteresis loop due to the texturing of the sample.

Thus, neglecting the easy-axis distributions results in incorrectly big $H_{E}^{\mathrm{ac}} / H_{e b}^{\mathrm{dc}}$ ratios. This was checked here by deriving $H_{E}^{\text {ac }}$ and comparing it with $H_{e b}^{\mathrm{dc}}$ for an off-aligned system that presents easy-axis distributions assuming the same anisotropy parameters as those for the corresponding singledomain case discussed above. The FM easy axes were considered to be almost randomly distributed (most populated $\phi_{F M}$ of $66^{\circ}$ and standard deviation of $150^{\circ}$ ) in the film's plane, and the AF ones randomly distributed in an in-plane cone with a half-apex angle of $55^{\circ}$ with respect to the dc field direction. The $H_{E}^{\mathrm{ac}} / H_{e b}^{\mathrm{dc}}$ ratio thus estimated is as high as 5.3 instead of 1.0 .

In order to verify the validity of the effect of the easy-axis distribution on the estimation of the anisotropy parameters on a real sample, we performed a very straightforward experiment. First, the easy-axis hysteresis loop as well as $M_{F M} / \chi_{t}=192$ Oe were measured by means of alternating gradient field magnetometer and superconducting quantum interference device, respectively, on an $\operatorname{IrMn}(15 \mathrm{~nm}) / \mathrm{Co}(5 \mathrm{~nm})$ rectangular-shaped sample. The latter has been deposited by magnetron sputtering and annealed for $15 \mathrm{~min}$ at $200{ }^{\circ} \mathrm{C}$ in $\mathrm{Ar}$ atmosphere with magnetic field of $1.6 \mathrm{kOe}$ applied in the plane of the films. Its $H_{E}$ has been recently estimated through both FMR and dc magnetometry ${ }^{14,30}$ and very similar values, $J_{E}^{\mathrm{FMR}} \simeq 1.1 J_{E}^{\mathrm{dc}}$, were obtained.

Using the same easy-axis distributions and anisotropy parameters employed in the magnetization curve simulations, ${ }^{14}$ we obtained $M_{F M} / \chi_{t}=173$ Oe, very close to the experimental value. That is, the three techniques gave approximately the same exchange-coupling strengths. This consistency is due to the relatively high magnetic texturing of our sample (FM and AF easy-axis distributions with small standard deviations of $6^{\circ}$ and $7^{\circ}$, respectively), which allowed us to use a single exchange-coupled FM/AF pair model to interpret the experiment.

Subsequently, a new sample was created by using a simple "top-down approach" - the same piece of film was cut along its easy direction into three equal pieces and two of 
them were set to form $+30^{\circ}$ and $-30^{\circ}$ with the other. The corresponding easy-axis loop is shown in Fig. 2 together with that for the original sample. Here, it is worth mentioning that the respective hysteresis loops of each of the three pieces measured before arranging the new sample (not shown) were practically the same as that of the original film, confirming that the cut has not changed their magnetic characteristics.

Here, $H_{c}$ and $H_{e b}^{\mathrm{dc}}$ of the new, less-textured sample decreased $24 \%$ and $8 \%$, respectively, while $M_{F M} / \chi_{t}$ increased $89 \%$. Although $H_{E}$ (i.e., the exchange-coupling field at the $\mathrm{FM} / \mathrm{AF}$ interface) is obviously one and the same for both samples, the $H_{E}^{\mathrm{ac}} / H_{e b}^{\mathrm{dc}}$ ratio, estimated by means of Eq. (6) and using $H_{U}=H_{c}$, was artificially increased 2.2 times. Certainly, if another sample is set with higher than $30^{\circ}$ maxi- mum angular deviation in the arrangement of the pieces, this value will be even greater.

In conclusion, we demonstrate that the great difference between the FM/AF exchange couplings, obtained by means of interpretation of magnetic susceptibility and dc magnetometry measurements, could have its origin in neglecting the FM and/or AF easy-axis distributions or underestimating the FM uniaxial anisotropy.

The authors thank J. Nogués and G. Salazar-Alvarez for critical reading of this Brief Report and useful discussions. J.G. acknowledges the sabbatical funding (No. SAB20060193) from the Spanish Ministry of Science and Education. This work has also been supported by the Brazilian agencies CNPq and FAPERGS.
${ }^{1}$ W. H. Meiklejohn and C. P. Bean, Phys. Rev. 102, 1413 (1956); 105, 904 (1957).

${ }^{2}$ W. H. Meiklejohn, J. Appl. Phys. 33, 1328 (1962).

${ }^{3}$ B. H. Miller and E. Dan Dahlberg, Appl. Phys. Lett. 69, 3932 (1996).

${ }^{4}$ V. Ström, B. J. Jönsson, K. V. Rao, and E. D. Dahlberg, J. Appl. Phys. 81, 5003 (1997).

${ }^{5}$ R. D. McMichael, M. D. Stiles, P. J. Chen, and W. F. Egelhoff, Jr., Phys. Rev. B 58, 8605 (1998).

${ }^{6}$ T. Pokhil, S. Mao, and A. Mack, J. Appl. Phys. 85, 4916 (1999).

${ }^{7}$ E. D. Dahlberg, B. Miller, B. Hill, B. J. Jönsson, V. Ström, K. V. Rao, J. Nogués, and Ivan K. Schuller, J. Appl. Phys. 83, 6893 (1998).

${ }^{8}$ P. Miltényi, M. Gruyters, G. Güntherodt, J. Nogués, and Ivan K. Schuller, Phys. Rev. B 59, 3333 (1999).

${ }^{9}$ H. Xi, R. M. White, and S. M. Rezende, Phys. Rev. B 60, 14837 (1999).

${ }^{10}$ J. R. Fermin, M. A. Lucena, A. Azevedo, F. M. de Aguiar, and S. M. Rezende, J. Appl. Phys. 87, 6421 (2000).

${ }^{11}$ R. L. Rodríguez-Suárez, L. H. Vilela Leão, F. M. de Aguiar, S. M. Rezende, and A. Azevedo, J. Appl. Phys. 94, 4544 (2003).

${ }^{12}$ J. Geshev, Phys. Rev. B 62, 5627 (2000).

${ }^{13}$ J. Geshev, L. G. Pereira, and J. E. Schmidt, Phys. Rev. B 64, 184411 (2001).

${ }^{14}$ J. Geshev, S. Nicolodi, L. G. Pereira, L. C. C. M. Nagamine, J. E. Schmidt, C. Deranlot, F. Petroff, R. L. Rodríguez-Suárez, and A. Azevedo, Phys. Rev. B 75, 214402 (2007).

${ }^{15}$ J. Åkerman, V. Ström, K. V. Rao, and E. D. Dahlberg, Phys. Rev. B 76, 144416 (2007).

${ }^{16}$ J. Geshev, L. G. Pereira, J. E. Schmidt, L. C. C. M. Nagamine, E. B. Saitovitch, and F. Pelegrini, Phys. Rev. B 67, 132401
(2003).

${ }^{17}$ J. Nogués and Ivan K. Schuller, J. Magn. Magn. Mater. 192, 203 (1999);.

${ }^{18}$ A. E. Berkowitz and K. Takano, J. Magn. Magn. Mater. 200, 552 (1999).

${ }^{19}$ J. Nogués, J. Sort, V. Langlais, V. Skumryev, S. Suriñach, J. S. Muñoz, and M. D. Baró, Phys. Rep. 422, 65 (2005).

${ }^{20}$ L. Spinu, IEEE Trans. Magn. 39, 2516 (2003).

${ }^{21}$ D. Mauri, H. C. Siegmann, P. S. Bagus, and E. Kay, J. Appl. Phys. 62, 3047 (1987).

${ }^{22}$ J. Camarero, J. Sort, A. Hoffmann, J. M. García-Martín, B. Dieny, R. Miranda, and J. Nogués, Phys. Rev. Lett. 95, 057204 (2005).

${ }^{23}$ R. W. Chantrell, A. Hoare, D. Melville, H. J. Lutke-Stetzkamp, and S. Methfessel, IEEE Trans. Magn. 25, 4216 (1989).

${ }^{24}$ J. Geshev, L. G. Pereira, and J. E. Schmidt, Phys. Rev. B 66, 134432 (2002).

${ }^{25}$ Since the total FM magnetization is the weighted average of the individual (for each pair of FM/AF domains) magnetizations, its derivative with respect to the field is the weighted average of the individual derivatives, permitting us to obtain $\chi_{t}$ numerically for textured FM/AF systems also.

${ }^{26}$ S. Chikazumi, Physics of Magnetism (Wiley, New York, 1964), p. 281.

${ }^{27}$ H. Xi and R. M. White, J. Appl. Phys. 86, 5169 (1999).

${ }^{28}$ A. Layadi, J. Appl. Phys. 90, 4651 (2001).

${ }^{29}$ C. Driemeier, L. C. C. M. Nagamine, J. E. Schmidt, and J. Geshev, J. Magn. Magn. Mater. 272-276, e811 (2004).

${ }^{30}$ S. Nicolodi, L. C. C. M. Nagamine, A. D. C. Viegas, J. E. Schmidt, L. G. Pereira, C. Deranlot, F. Petroff, and J. Geshev, J. Magn. Magn. Mater. 316, e97 (2007). 